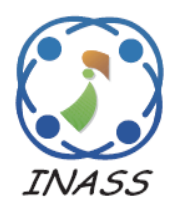

\title{
Convergence and Stability Analysis of Spline Adaptive Filtering based on Adaptive Averaging Step-size Normalized Least Mean Square Algorithm
}

\author{
Adisorn Saenmuang $^{1 *} \quad$ Suchada Sitjongsataporn $^{2}$ \\ ${ }^{I}$ The Electrical Engineering Graduate Program, Faculty of Engineering, \\ Mahanakorn University of Technology, Bangkok Thailand 10530 \\ ${ }^{2}$ Department of Electronic Engineering, Mahanakorn Institute of Innovation, Faculty of Engineering, \\ Mahanakorn University of Technology, Bangkok Thailand 10530 \\ * Corresponding author's Email: adisorn.sa41@gmail.com
}

\begin{abstract}
This paper presents a normalized version of least mean square algorithm with an adaptive averaging stepsize approach for spline adaptive filter. The use of an adaptive averaging step-size mechanism is to modify on autocorrelation between previous and present estimate error of system for updating step-size parameter. For achieving fast convergence, the proposed spline adaptive filter is combined with adaptive averaging step-size scheme and normalized version of least mean square approach. The convergence analysis and stability properties are accomplished. Simulation results of experiments depict that the trajectories of step-size parameters of the proposed algorithm converge to their own equilibria in spite of large variations in initial step-size settings. Proposed algorithm demonstrates more robust performance in mean square error and fast convergence compared with the conventional spline adaptive filter.
\end{abstract}

Keywords: Spline adaptive filtering, Nonlinear network, Normalized least mean square algorithm.

\section{Introduction}

Linear adaptive filtering is widely used for the solution to simply determine with the suitable constraint [1]. In opposition, many practical models necessitate the use of nonlinear adaptive filter in which nonlinear problem has more attention than linear operating system [2].

Spline adaptive filtering (SAF) based on least mean square (LMS) algorithm is a class of nonlinear adaptive filtering introduced in [2-4] with the low computation complexity and modelled in non-linear identification systems [5]. SAF is fabricated by adaptive linear finite impulse response (FIR) filtering followed by an adaptive lookup table (LUT).

Nonlinearity SAF structure using lookup table adjustment with the control points has been proposed in [3]. Sandwich SAF model in forms of cascade SAF architecture consists of a class of nonlinear models as linear-nonlinear-linear and nonlinearlinear-nonlinear models based on SAF structure, which can optimize using gradient-based condition in many conventional solutions of application [6, 7].

For nonlinear system identification, the authors in [8-10] conducted the normalized version of LMS (NLMS) scheme using the gradient-based criterion to improve performance of adaptive filtering, while the authors in [10] induced the potential performance in the case of infinite impulse response.

Against the impulsive noise, a set-membership scheme with the normalized version of least $M$ estimate algorithm has been developed in [11]. Simulation results depict that it can attain the achievable convergence rate. In [12], a sign normalized Wiener SAF is proposed in order to enhance the convergence by minimizing the absolute value of a posteriori error. 


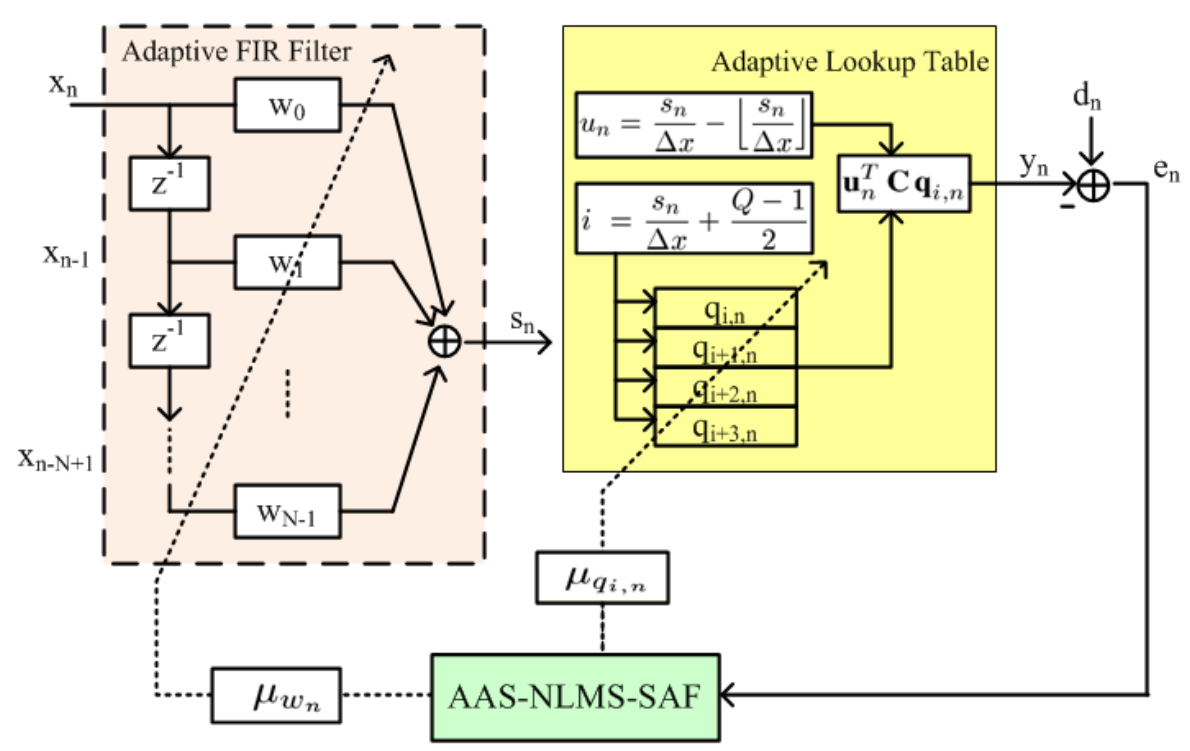

Figure.1 Linear-nonlinear network of AAS-NLMS-SAF structure.

To establish the well tracking and fast convergence, the adaptive step-size mechanism based on LMS algorithm is a well-known approach with effective solution for achieving the convergence in linear adaptive filtering [13], [14]. In [15], an idea of time averaging applied on adaptive step-size algorithm for beam forming has been modified with the low computation. In [16], a low complexity stepsize method by utilizing an approximate of autocorrelation error between present and previous estimate error is rearranged adaptively.

In this paper, we introduce a low complexity adaptive step-size approach based on normalized LMS algorithm in the SAF structure to achieve the fast convergence. In especial, we focus on the convergence analysis and mean square error performance of proposed algorithm.

This paper is arranged in this following. Section II describes briefly about SAF based on LMS. Section III proposes an adaptive averaging step-size algorithm for both the weight vectors of adaptive linear FIR filtering and interpolating control points of adaptive LUT by the minimizing cost function. Section IV shows the convergence and stability analysis of proposed algorithm. Experiment results and conclusion is in Section V and VI, respectively.

Notations are used through this paper. Operator (.) $)^{T}$ is the operation of transposition. Matrices and vectors are in bold uppercase and lowercase, respectively.

\section{Spline adaptive filtering}

The structure of spline adaptive filter (SAF), namely linear-nonlinear network, shows in Fig. 1. This network consists of linear and nonlinear part which is the linear part used the adaptive finite impulse response (FIR) filter and nonlinear part is an adaptive lookup table (LUT) with the spline interpolation network [2].

Consider a desired signal $d_{n}$ as

$$
d_{n}=y_{n}+e_{n}
$$

where $y_{n}$ is the spline adaptive filtering (SAF) output and $e_{n}$ is the system error.

The output of adaptive FIR filter $\boldsymbol{s}_{n}$ can be defined as

$$
\boldsymbol{s}_{n}=\boldsymbol{w}_{n}^{T} \mathbf{x}_{n}
$$

where $\mathbf{w}_{n}$ is the adaptive tap-weight vector and $\mathbf{x}_{n}$ is the input vector as

$$
\begin{aligned}
\mathbf{w}_{n} & =\left[\begin{array}{llll}
\mathrm{w}_{0} & \mathrm{w}_{1} & \ldots & \mathrm{w}_{N-1}
\end{array}\right], \\
\mathbf{x}_{n} & =\left[\begin{array}{llll}
x_{n} & x_{n-1} & \ldots & x_{n-N+1}
\end{array}\right],
\end{aligned}
$$

Following [17], the output of SAF is defined as

$$
\begin{aligned}
& y_{n}=\mathbf{u}_{n}^{T} \mathbf{C} \mathbf{q}_{i, n}, \\
& \mathbf{u}_{n}=\left[u_{n}^{3}, u_{n}^{2}, u_{n}, 1\right]^{T}
\end{aligned}
$$

where $\mathbf{q}_{i, n}$ is the control points vector as 


$$
\mathbf{q}_{i, n}=\left[q_{i, n} q_{i+1, n} q_{i+2, n} q_{i+3, n}\right]^{T}
$$

Local parameter $u_{n}$ and index $i$ are defined as [18]

$$
u_{n}=\frac{s_{n}}{\Delta x}-\left[\frac{s_{n}}{\Delta x}\right], \quad i=\left[\frac{s_{n}}{\Delta x}\right]+\frac{Q-1}{2} .
$$

where $\Delta x$ is the uniform space between twoadjacent control points. $Q$ is the number of control point, and $\lfloor\cdot]$ is floor operator. The parameter $s_{n}$ is concerned with a nonlinear activation function using the span index $i$ and the local parameter $u$, where $u \in[0,1]$. Spline basis matrix $\mathbf{C}$ is described in [2].

By minimizing cost function in the least mean square algorithm (LMS), we have [2]

$$
J\left(\mathbf{w}_{n}, \mathbf{q}_{i, n}\right)=\frac{1}{2} \min _{w_{n}}\left\{\left|e_{n}^{2}\right|\right\},
$$

where $e_{n}$ is a priori estimation error $e_{n}$ that arises from the model as

$$
e_{n}=d_{n}-y_{n}=d_{n}-\mathbf{u}_{n}^{T} \mathbf{C} \mathbf{q}_{i, n}
$$

Hence, the adaptive tap-weight $\mathbf{w}_{n}$ and $\mathbf{q}_{i, n}$ vectors take the specific form as

$$
\begin{aligned}
& \mathbf{w}_{n+1}=\mathbf{w}_{n}-\mu_{w} \frac{\partial J\left(\mathbf{w}_{n}, \mathbf{q}_{i, n}\right)}{\partial_{\mathbf{w}_{n}}} \\
& \mathbf{q}_{i, n+1}=\mathbf{q}_{i, n}-\mu_{q} \frac{\partial J\left(\mathbf{w}_{n}, \mathbf{q}_{i, n}\right)}{\partial \mathbf{q}_{i, n}},
\end{aligned}
$$

where $\mu_{w}$ and $\mu_{q}$ are the step-size parameters.

Then, the gradient of the cost function in Eq. (6) is necessarily evaluated with respect to (w.r.t) the adaptive tap-weight $\mathbf{w}_{n}$ and $\mathbf{q}_{i, n}$ vectors using the chain rule as

$$
\begin{aligned}
\frac{\partial J\left(\mathbf{w}_{n}, \mathbf{q}_{i, n}\right)}{\partial \mathbf{w}_{n}} & =-e_{n} \frac{\partial y_{n}}{\partial \mathbf{u}_{n}} \frac{\partial \mathbf{u}_{n}}{\partial \mathbf{s}_{n}} \frac{\partial \mathbf{s}_{n}}{\partial \mathbf{w}_{n}} \\
& =\frac{-e_{n}}{\Delta x} \mathbf{u}_{n}^{\prime} \mathbf{C} \mathbf{q}_{i, n} \mathbf{x}_{n}
\end{aligned}
$$

where the derivative of $\mathbf{u}_{\boldsymbol{n}}$ is given as

$$
\mathbf{u}_{n}^{\prime}=\left[3 u_{n}^{2}, 2 u_{n}, 1,0\right] \text {, }
$$

and

$$
\begin{aligned}
\frac{\partial J\left(\mathbf{w}_{n}, \mathbf{q}_{i, n}\right)}{\partial \mathbf{q}_{i, n}} & =-e_{n} \frac{\partial y_{n}}{\partial \mathbf{u}_{n}} \frac{\partial \mathbf{u}_{n}}{\partial \mathbf{s}_{n}} \frac{\partial \mathbf{s}_{n}}{\partial \mathbf{q}_{i, n}} \\
& =-e_{n} \mathbf{C}^{T} \mathbf{u}_{n} .
\end{aligned}
$$

According to Eqs. (10) and (12), the tap-weight LMS $\mathbf{w}_{n}$ and $\mathbf{q}_{i, n}$ vectors in the recursion form can be represented as [2]

$$
\begin{aligned}
& \mathbf{w}_{n+1}=\mathbf{w}_{n}+\mu_{w} \mathbf{u}_{n}^{\prime T} \mathbf{C} \mathbf{q}_{i, n} \mathbf{x}_{n} e_{n}, \\
& \mathbf{q}_{i, n+1}=\mathbf{q}_{i, n}+\mu_{q} \mathbf{C}^{T} \mathbf{u}_{n} e_{n}
\end{aligned}
$$

where $\mu_{w}$ and $\mu_{q}$ are the fixed step-size parameters for tap-weight $\mathbf{w}_{n}$ and for the control points $\mathbf{q}_{i, n}$, which incorporate with the other constant.

\section{Proposed adaptive averaging step-size normalized least mean square algorithm for spline adaptive filtering}

Following [11], the minimized cost function of normalized least mean square algorithm for SAF is expressed as

$$
\tilde{J}\left(\mathbf{w}_{n}, \mathbf{q}_{i, n}\right)=\frac{1}{2} \min _{w_{n}}\left\{\left(\mathbf{u}_{n}^{T} \mathbf{u}_{n}\right)^{-1}\left|e_{n}^{2}\right|\right\}
$$

where $e_{n}$ is defined in (7).

And the update tap-weight estimated vector $\mathbf{w}_{n}$ at symbol $n$ can be expressed by

$$
\mathbf{w}_{n+1}=\mathbf{w}_{n}-\mu_{w_{n}} \frac{\partial \tilde{J}\left(\mathbf{w}_{n}, \mathbf{q}_{i, n}\right)}{\partial_{\mathbf{w}_{n}}} .
$$

By differentiating the cost function in Eq. (15) w.r.t $\boldsymbol{w}_{n}$ with the chain rule, that is

$$
\begin{gathered}
\frac{\partial \tilde{J}\left(\mathbf{w}_{n}, \mathbf{q}_{1, n}\right)}{\partial \mathbf{w}_{n}}=\left(\mathbf{u}_{n}^{T} \mathbf{u}_{n}\right)^{-1}\left\{-e_{n} \frac{\partial y_{n}}{\partial \mathbf{u}_{n}} \frac{\partial \mathbf{u}_{n}}{\partial \mathbf{s}_{n}} \frac{\partial \mathbf{s}_{n}}{\partial \mathbf{w}_{n}}\right\} \\
=\left(\mathbf{u}_{n}^{T} \mathbf{u}_{n}\right)^{-1}\left\{\frac{-e_{n}}{\Delta x} \mathbf{u}_{n}^{\prime} \mathbf{C} \mathbf{q}_{i, n} \mathbf{x}_{n}\right\}
\end{gathered}
$$

Finally, we introduce the proposed tap-weight estimated vector $\boldsymbol{w}_{\boldsymbol{n}}$ of adaptive FIR filter based on normalized least mean square algorithm obtained by

$$
\therefore \mathbf{w}_{n+1}=\mathbf{w}_{n}+\mu_{w_{n}} \frac{\mathbf{u}_{n}^{\prime} \mathbf{C} \mathbf{q}_{i, n} \mathbf{x}_{n} e_{n}}{\Delta x \mathbf{u}_{n}^{T} \mathbf{u}_{n}}
$$

where $\mu_{w_{n}}$ is the adaptive step-size parameter for learning rate of linear part of SAF structure.

Similarly, the update estimated control points vector $\mathbf{q}_{i, n}$ at symbol $n$ can be obtained by

$$
\mathbf{q}_{i, n+1}=\mathbf{q}_{i, n}-\mu_{q_{i, n}} \frac{\partial \tilde{J}\left(\mathbf{w}_{n}, \mathbf{q}_{i, n}\right)}{\partial \mathbf{q}_{i, n}}
$$


Hence, the gradient of the cost function in Eq. (15) w.r.t $\mathbf{q}_{i, n}$ using the chain rule is defined by

$$
\begin{aligned}
\frac{\partial \tilde{J}\left(\mathbf{w}_{n}, \mathbf{q}_{i, n}\right)}{\partial \mathbf{q}_{i, n}} & =\left(\mathbf{u}_{n}^{T} \mathbf{u}_{n}\right)^{-1}\left\{-e_{n} \frac{\partial y_{n}}{\partial \mathbf{u}_{n}} \frac{\partial \mathbf{u}_{n}}{\partial \mathbf{s}_{n}} \frac{\partial \mathbf{s}_{n}}{\partial \mathbf{q}_{i, n}}\right\} \\
& =\left(\mathbf{u}_{n}^{T} \mathbf{u}_{n}\right)^{-1}\left\{-e_{n} \mathbf{C}^{T} \mathbf{u}_{n}\right\}
\end{aligned}
$$

Therefore, we present the control points vector $\mathbf{q}_{i, n}$ based on normalized least mean square algorithm of nonlinear network in the adaptive lookup table as

$$
\therefore \mathbf{q}_{i, n+1}=\mathbf{q}_{i, n}+\mu_{q_{n}} \frac{\mathbf{c}^{T} \mathbf{u}_{n} e_{n}}{\mathbf{u}_{n}^{T} \mathbf{u}_{n}}
$$

where $\mu_{q_{n}}$ is the adaptive step-size parameter for nonlinear part of SAF structure.

\subsection{Adaptive averaging step-size algorithm for spline adaptive filtering}

The main objective of adaptive averaging stepsize mechanism is to improve as follows. Following [17], if the estimate error is far off the optimal value, the step-size parameter will be increased. Meanwhile, the estimate error is near the optimum, the step-size parameter will be decreased automatically.

The proposed idea is to average step-size parameter with autocorrelation of previous and present estimate error of network system for update $\mu_{w_{n}}$ and $\mu_{q_{n}}$ adaptively.

Therefore, we modify the adaptive averaging step-size $\mu_{w_{n}}$ of tap-weight $\mathbf{w}_{n}$ vector concerning with the estimation of an averaging of autocorrelation $\left\{e_{n-1}^{*} e_{n}\right\}$ as

$$
\begin{aligned}
\mu_{w_{n}} & =\alpha_{w} \cdot \mu_{w_{n-1}}+\beta_{w} \cdot\left|\xi_{n}\right|^{2}, \\
\xi_{n} & =\gamma \cdot \xi_{n-1}+(1-\gamma)\left\{e_{n-1}^{*} e_{n}\right\},
\end{aligned}
$$

where $\beta_{w}$ is a scaled variable for prediction error, $\gamma$ is close to 1 and $0<\alpha_{w}<1$.

We note that there are two reasons related with $\xi_{n}$ are as follows. First, the autocorrelation of error is generally measured for optimal performance. Second, the uncorrelated noise sequence is rejected on the update step-size mechanism.

\subsection{Modified adaptive step-size algorithm}

Following [19], the learning rate of step-size is controlled by squared estimate error. If an error is large, the step-size parameter will increase. While a small error will yield misadjustment with the decreased step-size value. Therefore, the step-size parameter $\mu_{q_{n}}$ of control points vector $\mathbf{q}_{i, n}$ is

$$
\mu_{q_{n}}=\alpha_{q} \cdot \mu_{q_{, n-1}}+\beta_{q} \cdot\left|e_{n}\right|^{2},
$$

where $0<\alpha_{q}<1, \beta_{q}>0$ and a priori estimate error $e_{n}$ is given in Eq. (7).

Summary of proposed adaptive averaging stepsize mechanism based on the normalized version of least mean square algorithm for spline adaptive filter (AAS-NLMS-SAF) is shown in Table 1.

\section{Convergence and stability analysis}

In order to achieve optimal performance, we determine an adaptive leaning rate that minimizes the instantaneous output error of filter by performing Taylor series expansion of error $e_{n}$. The approach intends to the optimal learning rate to ensure the convergence at the steady-state.

\subsection{Convergence analysis of proposed algorithm}

Convergence properties of adaptive tap-weight $\mathbf{w}_{n}$ vector can be determined by using Taylor series expansion of estimate error $e_{n}$ as [2]

$$
e_{n+1} \simeq e_{n}+\frac{\partial e_{n}}{\partial \mathbf{w}_{n}} \cdot \Delta \mathbf{w}_{n}
$$

where an estimate error $e_{n}$ is given as

$$
e_{n}=d_{n}-\mathbf{u}_{n}^{T} \cdot \mathbf{C} \cdot \mathbf{q}_{i, n} \cdot
$$

Differentiating $e_{n}$ in Eq. (26) w.r.t $\mathbf{w}_{n}$ with the chain rule, we get

$$
\frac{\partial e_{n}}{\partial \mathbf{w}_{\boldsymbol{n}}}=\frac{-\mathbf{u}_{n}^{\prime} \cdot \mathbf{C} \cdot \mathbf{q}_{i, n} \cdot \mathbf{x}_{\boldsymbol{n}}}{\Delta x \cdot\left(\mathbf{u}_{n}^{T} \cdot \mathbf{u}_{n}\right)} .
$$

where $\mathbf{u}_{n}^{\prime}$ is given in Eq. (11).

From Eq. (18), we have the change of $\mathbf{w}_{n}$ as

$$
\Delta w_{n}=\mathbf{w}_{n+1}-\mathbf{w}_{n}=\frac{\mu_{w_{n}} \cdot \mathbf{u}_{n}^{\prime} \cdot \mathbf{C} \cdot \mathbf{q}_{i, n} \cdot \mathbf{x}_{n} \cdot e_{n}}{\Delta x \cdot\left(\mathbf{u}_{n}^{T} \cdot \mathbf{u}_{n}\right)}
$$

By substituting (27) and (28) into (25), we arrive at

$$
e_{n+1}=e_{n}-\frac{\mu_{w_{n}}}{\Delta x}\left(\frac{\emptyset_{n} \cdot \mathbf{x}_{n}}{\mathbf{u}_{n}^{T} \cdot \mathbf{u}_{n}}\right)\left(\frac{\emptyset_{n} \cdot \mathbf{x}_{n} \cdot e_{n}}{\Delta x \cdot \mathbf{u}_{n}^{T} \cdot \mathbf{u}_{n}}\right) .
$$

where $\emptyset_{n}$ is given by

$$
\emptyset_{n}=\mathbf{u}_{n}^{\prime} \cdot \mathbf{C} \cdot \mathbf{q}_{i, n} .
$$


Table 1. Proposed spline adaptive filtering based on the adaptive averaging step-size normalized least mean square algorithm (AAS-NLMS-SAF)

Initialize $: \mathbf{w}(0)=\varphi_{w^{*}}\left[\begin{array}{llll}1 & 0 & \ldots & 0\end{array}\right]^{T}, \mathbf{q}(0)=\left[\begin{array}{llll}1 & 0 & \ldots & 0\end{array}\right]^{T}$,

$\mathbf{C}=\frac{1}{2}\left[\begin{array}{rrrr}-1 & 3 & -3 & 1 \\ 2 & -5 & 4 & -1 \\ -1 & 0 & 1 & 0 \\ 0 & 2 & 0 & 0\end{array}\right]$

for $n=0,1,2, \ldots, N-1$.

1) Calculate the output of adaptive FIR filter $s_{n}$

$$
\boldsymbol{s}_{n}=\boldsymbol{w}_{n}^{T} \mathbf{x}_{n},
$$

2) Compute the local parameter $u_{n}$ and index $i$ as

$$
\begin{gathered}
u_{n}=\frac{s_{n}}{\Delta x}-\left[\frac{s_{n}}{\Delta x}\right] \\
i=\left[\frac{s_{n}}{\Delta x}\right]+\frac{Q-1}{2} .
\end{gathered}
$$

3) Calculate the error $e_{n}$ as

$$
e_{n}=d_{n}-\mathbf{u}_{n}^{T} \mathbf{C} \mathbf{q}_{i, n}
$$

4) Compute the adaptive averaging step-size $\mu_{w_{n}}$ of $\mathbf{w}_{n}$

$$
\begin{aligned}
\mu_{w_{n}} & =\alpha_{w} \cdot \mu_{w_{n-1}}+\beta_{w} \cdot\left|\xi_{n}\right|^{2}, \\
\xi_{n} & =\gamma \cdot \xi_{n-1}+(1-\gamma)\left\{e_{n-1}^{*} e_{n}\right\},
\end{aligned}
$$

5) Calculate the modified step-size $\mu_{q_{i, n}}$ of $q_{i, n}$

$$
\mu_{q_{n}}=\alpha_{q} \cdot \mu_{q_{n-1}}+\beta_{q} \cdot\left|e_{n}\right|^{2}
$$

6) Determine the tap-weight vector $\mathbf{w}_{n}$ and the control points vector $\mathbf{q}_{i, n}$ as

$$
\begin{aligned}
& \mathbf{w}_{n+1}=\mathbf{w}_{n}+\mu_{w_{n}} \frac{\mathbf{u}_{n}^{\prime} \mathbf{C} \mathbf{q}_{i, n} \mathbf{x}_{n} e_{n}}{\Delta x \mathbf{u}_{n}^{T} \mathbf{u}_{n}}, \\
& \mathbf{q}_{i, n+1}=\mathbf{q}_{i, n}+\mu_{q_{n}} \frac{\mathbf{c}^{T} \mathbf{u}_{n} e_{n}}{\mathbf{u}_{n}^{T} \mathbf{u}_{n}}
\end{aligned}
$$

end

Therefore, the estimate error can be rewritten as

$$
\therefore e_{n+1}=\left[1-\frac{\mu_{w_{n}}}{(\Delta x)^{2}}\left(\frac{\phi_{n}^{2} \cdot\left\|\mathbf{x}_{n}\right\|^{2}}{\left(\mathbf{u}_{n}^{T} \cdot \mathbf{u}_{n}\right)^{2}}\right)\right] e_{n} .
$$

Taking the norm of both sides in (31), we have

$$
\left|e_{n+1}\right|=\left|1-\frac{\mu_{w_{n}}}{(\Delta x)^{2}}\left(\frac{\emptyset_{n}^{2} \cdot\left\|\mathbf{x}_{n}\right\|^{2}}{\mathbf{u}_{n}^{T} \cdot \mathbf{u}_{n}}\right)\right| \cdot\left|e_{n}\right|
$$

Therefore, the proposed step-size $\mu_{w_{n}}$ of tap-weight vector $\mathbf{w}_{n}$ in the adaptive FIR filter reaches

$$
\therefore \mu_{w_{n}} \simeq \frac{2\left(\mathbf{u}_{n}^{T} \cdot \mathbf{u}_{n}\right)^{2} \cdot(\Delta x)^{2}}{\emptyset_{n}^{2} \cdot\left\|\mathbf{x}_{n}\right\|^{2}}
$$

where we assume that $\left|e_{n+1}\right|<\left|e_{n}\right|$.

Similarly, we determine a bound on $\mu_{q_{n}}$ with the Taylor series expansion of estimate error $e_{n}$ as

$$
e_{n+1}=e_{n}+\frac{\partial e_{n}}{\partial \mathbf{q}_{i, n}} \cdot \Delta \mathbf{q}_{i, n}
$$

where the derivative of $e_{n}$ w.r.t $\mathbf{q}_{i, n}$ is given by

$$
\frac{\partial e_{n}}{\partial \mathbf{q}_{i, n}}=\frac{-\mathbf{c}^{T} \cdot \mathbf{u}_{n}}{\mathbf{u}_{n}^{T} \cdot \mathbf{u}_{n}},
$$

And From (21), we have the change of $\mathbf{q}_{i, n}$ as

$$
\Delta \mathbf{q}_{i, n}=\mu_{q_{n}} \cdot \frac{\mathbf{c}^{T} u_{n} e_{n}}{u_{n}^{T} u_{n}} .
$$

Hence, we substitute Eqs. (35) and (36) into Eq. (34), we have

$$
\therefore e_{n+1}=\left[1-\left(\mu_{q_{n}} \cdot \frac{\mathbf{c}^{T} \cdot \mathbf{u}_{n}}{\mathbf{u}_{n}^{T} \mathbf{u}_{n}}\right) \cdot\left(\frac{\mathbf{C}^{T} \mathbf{u}_{n}}{\mathbf{u}_{n}^{T} \mathbf{u}_{n}}\right)\right] e_{n}
$$

Taking the norm of both sides in Eq. (37), we get

$$
\left|e_{n+1}\right|=\left|1-\left(\mu_{q_{n}} \cdot \frac{\mathbf{c}^{T} \cdot \mathbf{u}_{n}}{\mathbf{u}_{n}^{T} \mathbf{u}_{n}}\right) \cdot\left(\frac{\mathbf{c}^{T} \mathbf{u}_{n}}{\mathbf{u}_{n}^{T} \mathbf{u}_{n}}\right)\right| \cdot\left|e_{n}\right| .
$$

Therefore, the adaptive learning rate $\mu_{q_{n}}$ becomes

$$
\therefore \mu_{q_{n}} \cong 2 \frac{\mathbf{u}_{n}^{T} \cdot \mathbf{u}_{n}}{\mathbf{C}^{T} \mathbf{C}}
$$

\subsection{Mean square error performance of proposed algorithm}

In this section, we consider the mean square error performance at steady-state in the derivation of excess mean square error (EMSE) of nonlinear adaptive FIR filter and the control points vector in the adaptive LUT.

Following [6], we determine the $\varepsilon_{n}$ is a priori error of system, $\varepsilon_{w_{n}}$ is a priori error concerned the tap-weight vector $\mathbf{w}_{n}$ and $\varepsilon_{q_{n}}$ is a priori error involved the control points vector $\mathbf{q}_{i, n}$.

To encourage the analysis, the proposed adaptive averaging step - size normalized least 
mean square (AAS - NLMS) algorithm is under a few assumptions.

Assumption 1: We consider that the noise sequence of system $\boldsymbol{\eta}_{n}$ is independent and identically distributed with variance of noise $\delta^{2}$ and zero mean.

Assumption 2: We consider that the noise sequence of system $\boldsymbol{\eta}_{n}$ is independent of $\mathbf{x}_{n}, \mathbf{s}_{n}, \varepsilon_{n}, \varepsilon_{w_{n}}$, and $\varepsilon_{q_{n}}$

Let us assume the estimate weight noise vector $\boldsymbol{\eta}_{w_{n}}$ concerned with the tap - weight vector $\mathbf{w}_{n}$ as

$$
\boldsymbol{\eta}_{w_{n}}=\mathbf{w}_{0}-\mathbf{w}_{n}
$$

where $\boldsymbol{\eta}_{w_{n}}=\left[\begin{array}{llll}\eta_{w_{0}} & \eta_{w_{1}} \ldots \eta_{w_{N-1}}\end{array}\right]$.

From Eq. (18), we can write the update weight noise vector $\boldsymbol{\eta}_{w_{n+1}}$ as

$$
\begin{aligned}
& \boldsymbol{\eta}_{w_{n+1}}=\boldsymbol{\eta}_{w_{n}}-\left(\mathbf{w}_{n+1}-\mathbf{w}_{n}\right) \\
& \boldsymbol{\eta}_{w_{n+1}}=\boldsymbol{\eta}_{w_{n}}-\frac{\mu_{w_{n}} \cdot \emptyset_{n} \cdot \mathbf{x}_{n} \cdot e_{n}}{\Delta x\left(\mathbf{u}_{n}^{T} \cdot \mathbf{u}_{n}\right)}
\end{aligned}
$$

where $\emptyset_{n}$ is given in Eq. (30).

To evaluate the square of update weight noise vector $\left\|\boldsymbol{\eta}_{w_{n}}\right\|^{2}$ of Eq. (41), we obtain

$$
\begin{gathered}
\left\|\boldsymbol{\eta}_{w_{n+1}}\right\|^{2}=\left\|\boldsymbol{\eta}_{w_{n}}\right\|^{2}-\boldsymbol{\eta}_{w_{n}} \cdot \frac{\mu_{w n}}{\Delta x} \cdot \frac{\emptyset_{n} \cdot \mathbf{x}_{n} \cdot e_{n}}{\left(\mathbf{u}_{n}^{T} \mathbf{u}_{n}\right)} \\
+\cdot \frac{\mu_{w n}^{2}}{(\Delta x)^{2}} \cdot \frac{\emptyset_{n}^{2} \cdot\left\|\mathbf{x}_{n}\right\|^{2} \cdot e_{n}^{2}}{\left(\mathbf{u}_{n}^{T} \mathbf{u}_{n}\right)^{2}}
\end{gathered}
$$

Assumption 3: We consider the condition necessary for the convergence of mean, that is

$$
\mathbf{E}\left\{\left\|\boldsymbol{\eta}_{w_{n+1}}\right\|^{2}\right\}=\mathbf{E}\left\{\left\|\boldsymbol{\eta}_{w_{n}}\right\|^{2}\right\} \text {, as } n \rightarrow \infty \text {. }
$$

From Assumption (3), the update $\boldsymbol{\eta}_{w_{n}}$ in (42) can be rewritten as

$$
\begin{aligned}
2 \boldsymbol{\eta}_{w_{n}} \cdot \frac{\mu_{w_{n}}}{\Delta x} \cdot \frac{\emptyset_{n} \cdot \mathbf{x}_{n} \cdot e_{n}}{\left(\mathbf{u}_{n}^{T} \cdot \mathbf{u}_{n}\right)} & =\frac{\mu_{w_{n}}^{2}}{(\Delta x)^{2}} \cdot \frac{\emptyset_{n}^{2} \cdot\left\|\mathbf{x}_{n}\right\|^{2} e_{n}^{2}}{\left(\mathbf{u}_{n}^{T} \cdot \mathbf{u}_{n}\right)^{2}} \\
2 \varepsilon_{w_{n}} \cdot e_{n} & =\frac{\mu_{w_{n}}}{\Delta x} \cdot \frac{\emptyset_{n} \cdot\left\|\mathbf{x}_{n}\right\|^{2} \cdot e_{n}^{2}}{\left(\mathbf{u}_{n}^{T} \cdot \mathbf{u}_{n}\right)},
\end{aligned}
$$

where $\varepsilon_{w_{n}}$ is given by

$$
\varepsilon_{w_{n}}=\boldsymbol{\eta}_{w_{n}} \mathbf{x}_{n}
$$

To redefine the a priori error of system $e_{n}$ as

$$
e_{n}=\varepsilon_{w_{n}}+\eta_{w_{n}}
$$

Taking the expectation onto the noise in Eqs. (44) and (45) with the condition at steady-state for $n$ closes to infinity, we get

$$
E\left\{\varepsilon_{w_{n}} \cdot e_{n}\right\}=E\left\{\varepsilon_{w_{n}}\left(\varepsilon_{w_{n}}+\eta_{w_{n}}\right)\right\} \simeq E\left\{\varepsilon_{w_{n}}^{2}\right\}
$$

and

$$
\begin{aligned}
E\left\{e_{n}^{2}\right\} & =E\left\{\left(\varepsilon_{w_{n}}+\eta_{w_{n}}\right)^{2}\right\} \\
& =E\left\{\varepsilon_{w_{n}}^{2}+2 \varepsilon_{w_{n}} \eta_{w_{n}}+\eta_{w}^{2}\right\} \\
& \simeq E\left\{\varepsilon_{w_{n}}^{2}+\xi_{w_{n}}^{2}\right\} .
\end{aligned}
$$

where $\xi_{w_{n}}^{2}$ is the minimum MSE involved with $\mathbf{w}_{n}$. Substituting Eqs. (46) and (47) into Eq. (43), we have

$$
\begin{gathered}
2 E\left\{\varepsilon_{w_{n}}^{2}\right\}=\frac{\mu_{w_{n}}}{\Delta x} \cdot \frac{\emptyset_{n} \cdot\left\|\mathbf{x}_{n}\right\|^{2}}{\left(\mathbf{u}_{n}^{T} \cdot \mathbf{u}_{n}\right)} \cdot \mathrm{E}\left\{\varepsilon_{w_{n}}^{2}+\xi_{w_{n}}^{2}\right\} \\
{\left[2-\frac{\mu_{w_{n}}}{\Delta x} \cdot \frac{\emptyset_{n} \cdot\left\|x_{n}\right\|^{2}}{\mathbf{u}_{n}^{T} \cdot \mathbf{u}_{n}}\right]\left\{E_{w_{n}^{2}}\right\}=\frac{\mu_{w_{n}}}{\Delta x} \cdot \frac{\emptyset_{n} \cdot\left\|\mathbf{x}_{n}\right\|^{2}}{\left(\mathbf{u}_{n}^{T} \cdot \mathbf{u}_{n}\right)} \cdot \mathrm{E}\left\{\xi_{w_{n}}^{2}\right\}} \\
E\left\{\varepsilon_{w_{n}}^{2}\right\}=\frac{\mu_{w_{n}} \emptyset_{n}\left\|\mathbf{x}_{n}\right\|^{2} E\left\{\xi_{w_{n}}^{2}\right\}}{2 \Delta x\left(\mathbf{u}_{n} \cdot \mathbf{u}_{n}\right)-\mu_{w_{n}} \cdot \emptyset_{n} \cdot\left\|\mathbf{x}_{n}\right\|^{2}}
\end{gathered}
$$

If $\mu_{w_{n}}$ is very small, we have

$$
\therefore \zeta_{w}=\mathrm{E}\left\{\mathcal{E}_{w_{n}}^{2}\right\} \cong \frac{\mu_{w_{n} \emptyset_{n}}\left\|\mathbf{x}_{n}\right\|^{2} \cdot E\left\{\xi_{w_{n}}^{2}\right\}}{2 \Delta x\left(\mathbf{u}_{n}^{T} \cdot \mathbf{u}_{n}\right)},
$$

where $\zeta_{w}$ is the excess MSE concerned with $\mathbf{w}_{n}$.

In a similar manner, we assume that the noise sequence of estimated weight noise vector $\boldsymbol{\eta}_{q_{n}}$ involved with the control points vector $\mathbf{q}_{i, n}$ as

$$
\boldsymbol{\eta}_{q_{n}}=\mathbf{q}_{0}-\mathbf{q}_{i, n}
$$

where $\boldsymbol{\eta}_{q_{n}}=\left[\begin{array}{llll}\eta_{q_{0}} & \eta_{q_{1}} \ldots & \eta_{q_{N-1}}\end{array}\right]$.

From Eq. (21), the update weight noise vector $\boldsymbol{\eta}_{q_{n}}$ can be expressed as

$$
\boldsymbol{\eta}_{q_{n+1}}=\boldsymbol{\eta}_{q_{n}}-\mu_{q_{n}} \frac{\mathbf{u}_{n}^{T} \cdot \mathbf{c} \cdot \boldsymbol{e}_{n}}{\left(\mathbf{u}_{n}^{T} \mathbf{u}_{n}\right)} .
$$

Then, we evaluate the square of noise vector $\left\|\boldsymbol{\eta}_{q_{n}}\right\|^{2}$ using Eq. (51), that is

$$
\left\|\boldsymbol{\eta}_{q_{n+1}}^{2}\right\|=\left\|\boldsymbol{\eta}_{q_{n}}^{2}\right\|-2 \boldsymbol{\eta}_{q_{n}} \mu_{q_{n}} \frac{\mathbf{u}_{n}^{T} \cdot \mathbf{C} \cdot e_{n}}{\left(\mathbf{u}_{n}^{T} \cdot \mathbf{u}_{n}\right)}+\frac{\mu_{q_{n}}^{2}\left\|\mathbf{u}_{n}^{T} \cdot \mathbf{C}\right\|^{2} e_{n}^{2}}{\left(\mathbf{u}_{n}^{T} \cdot \mathbf{u}_{n}\right)^{2}}
$$

Assumption 4: We regard that

$$
E\left\{\left\|\boldsymbol{\eta}_{q_{n+1}}\right\|^{2}\right\}=E\left\{\left\|\boldsymbol{\eta}_{q_{n}}\right\|^{2}\right\} ; \text { as } n \rightarrow \infty
$$

International Journal of Intelligent Engineering and Systems, Vol.13, No.2, 2020 
From Assumption (4), the update $\boldsymbol{\eta}_{q_{n}}$ in Eq. (52) can be calculated as

$$
\begin{array}{r}
2 \boldsymbol{\eta}_{q_{n}} \cdot \mu_{q_{n}} \cdot \frac{\boldsymbol{\mu}_{n}^{T} \cdot c_{n} \cdot e_{n}}{\left(\mathbf{u}_{n}^{T} \cdot \mathbf{u}_{n}\right)}=\frac{\mu_{q_{n}}^{2} \cdot\left\|\mathbf{u}^{T} \cdot \mathbf{c}\right\|^{2} \cdot e^{2 n}}{\left(\mathbf{u}_{n}^{T} \cdot \mathbf{u}_{n}\right)^{2}} \\
2 \mathcal{E}_{q_{n}} \cdot e_{n}=\mu_{q_{n}} \cdot \frac{\left\|\mathbf{u}^{T} \cdot \mathbf{c}\right\|^{2} \cdot e_{n}^{2}}{\left(\mathbf{u}_{n}^{T} \cdot \mathbf{u}_{n}\right)}
\end{array}
$$

where $\varepsilon_{q_{n}}$ is given as

$$
\mathcal{E}_{q_{n}}=\boldsymbol{\eta}_{q_{n}} \cdot \mathbf{u}_{n}^{T} \cdot \mathbf{C}
$$

So, we determine that the a priori error $e_{n}$ is involved with $\mathbf{q}_{i, n}$ as

$$
e_{n}=\varepsilon_{q_{n}}+\eta_{q_{n}}
$$

Taking the expectation into the noise in Eq. (53) and (55) at steady-state for $n \rightarrow \infty$, we have

$$
\begin{aligned}
& \mathrm{E}\left\{\varepsilon_{q_{n}} \cdot e_{n}\right\}=\mathrm{E}\left\{\varepsilon_{q_{n}} \cdot\left(\varepsilon_{q_{n}}+\eta_{q_{n}}\right)\right\} \simeq \mathrm{E}\left\{\varepsilon_{q_{n}}^{2}\right\} \\
& \mathrm{E}\left\{\varepsilon_{n}^{2}\right\}=\left\{\left(\varepsilon_{q_{n}}+\eta_{q_{n}}\right)^{2}\right\} \simeq \mathrm{E}\left\{\varepsilon_{q_{n}}^{2}+\xi_{q_{n}}^{2}\right\}
\end{aligned}
$$

where $\xi_{q_{n}}^{2}$ is the minimum MSE involved with $\mathbf{q}_{i, n}$. Replacing Eqs. (56) and (57) into Eq. (53), we get

$$
\begin{gathered}
2 \mathrm{E}\left\{\mathcal{E}_{q_{n}}^{2}\right\}=\mu_{\boldsymbol{q}_{n}} \cdot \frac{\left\|\mathbf{u}^{T} \cdot \mathbf{C}\right\|^{2}}{\left(\mathbf{u}_{n}^{T} \cdot \mathbf{u}_{n}\right)} \cdot \mathrm{E}\left\{\mathcal{E}_{q_{n}}^{2}+\xi_{q_{n}}^{2}\right\} \\
\mathrm{E}\left\{\varepsilon_{q_{n}}^{2}\right\}\left[2-\frac{\mu_{q_{n}} \cdot\left\|\mathbf{u}^{T} \cdot \mathbf{c}\right\|^{2}}{\left(\mathbf{u}_{n}^{T} \cdot \mathbf{u}_{n}\right)}\right]=\frac{\mu_{q_{n}} \cdot\left\|\mathbf{u}^{T} \cdot \mathbf{C}\right\|^{2} \cdot \mathrm{E}\left\{\xi_{q_{n}}^{2}\right\}}{\left(\mathbf{u}_{n}^{T} \cdot \mathbf{u}_{n}\right)} .
\end{gathered}
$$

If $\mu_{q_{n}}$ is very small, we get

$$
\therefore \zeta_{q} \simeq \mathrm{E}\left\{\mathcal{E}_{q_{n}}^{2}\right\}=\frac{\mu_{q_{n}} \cdot\left\|\mathbf{u}^{T} \cdot \mathbf{c}\right\|^{2} \cdot \mathbf{E}\left\{\xi_{q_{n}}^{2}\right\}}{2\left(\mathbf{u}_{n}^{T} \cdot \mathbf{u}_{n}\right)}
$$

where $\zeta_{q}$ is the excess MSE concerned with $\mathbf{q}_{i, n}$.

\section{Experimental results}

In this section, we provide the experimental tests in system identification by simulating the random process. The input coloured signal for all experiments comprises 5,000 samples of the signal generated in the system identification over 100 Monte Carlo trials by following [20].

$$
x_{n}=\alpha \cdot x_{n-1}+\sqrt{1-\alpha^{2}} \cdot \psi_{n},
$$

where $\psi_{n}$ denotes as a zero mean white Gaussian noise with unitary variance and $0.1 \leq \alpha<0.99$.

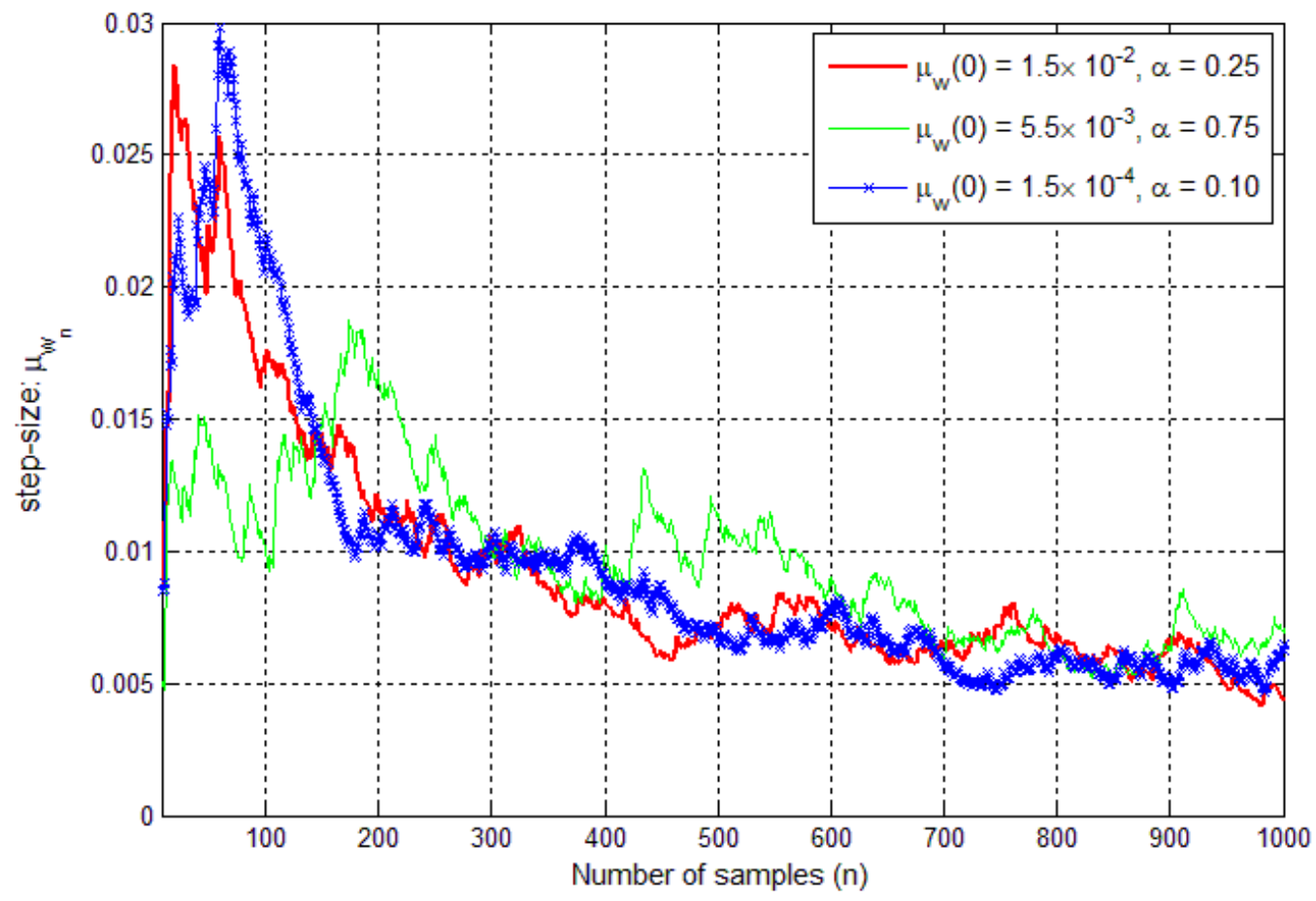

Figure. 2 Learning curves of $\mu_{w}(n)$ of tap-weight $\mathrm{w}_{n}$ vector of proposed AAS-NLMS-SAF algorithm with the different $\alpha=0.1,0.25,0.75$ and $\mathrm{SNR}=40 \mathrm{~dB}$ 


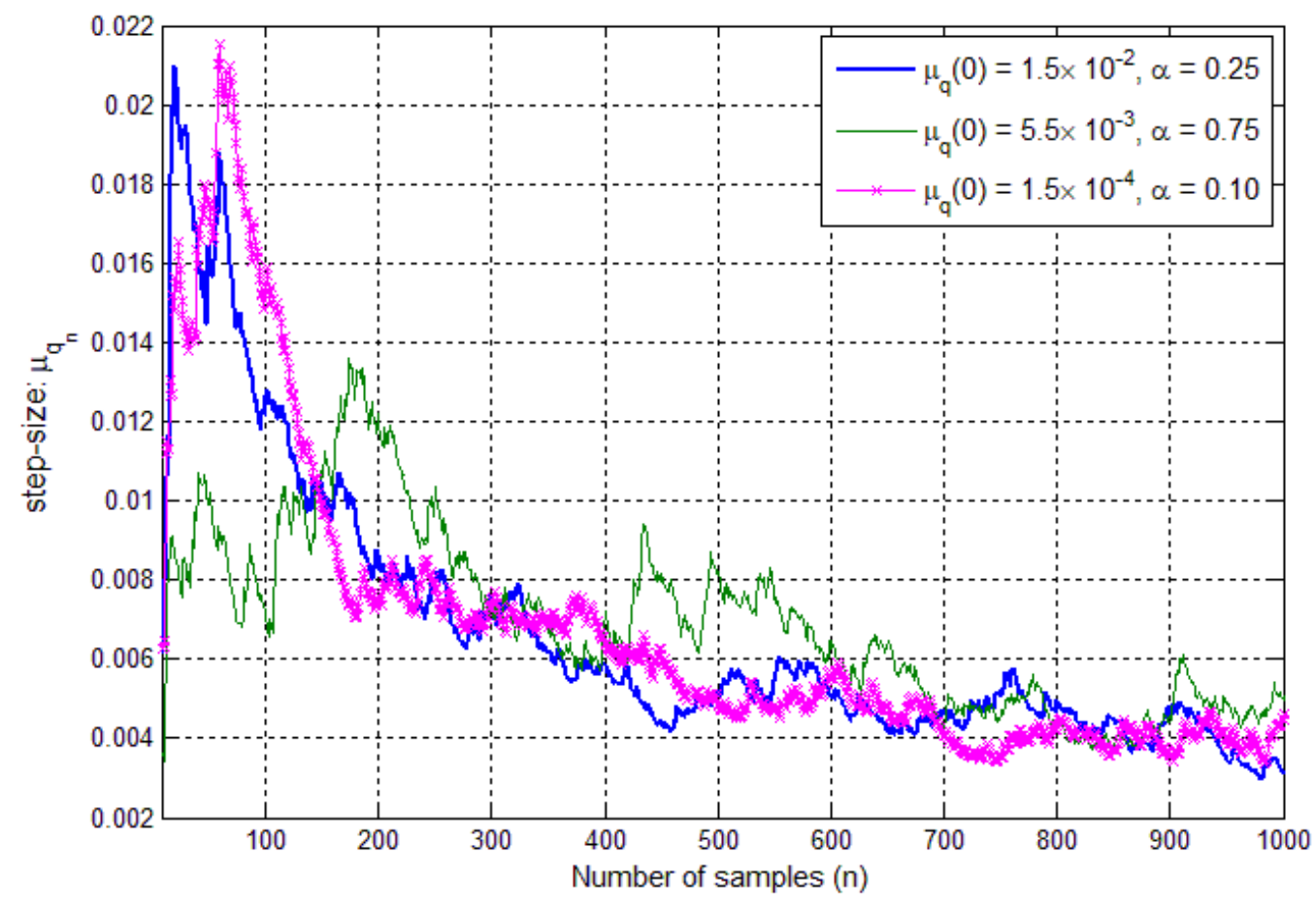

Figure.3 Learning curves of $\mu_{q}(n)$ of control points $\mathrm{q}_{i, n}$ vector of proposed AAS-NLMS-SAF algorithm the different $\alpha=0.1,0.25,0.75$ and $\mathrm{SNR}=40 \mathrm{~dB}$

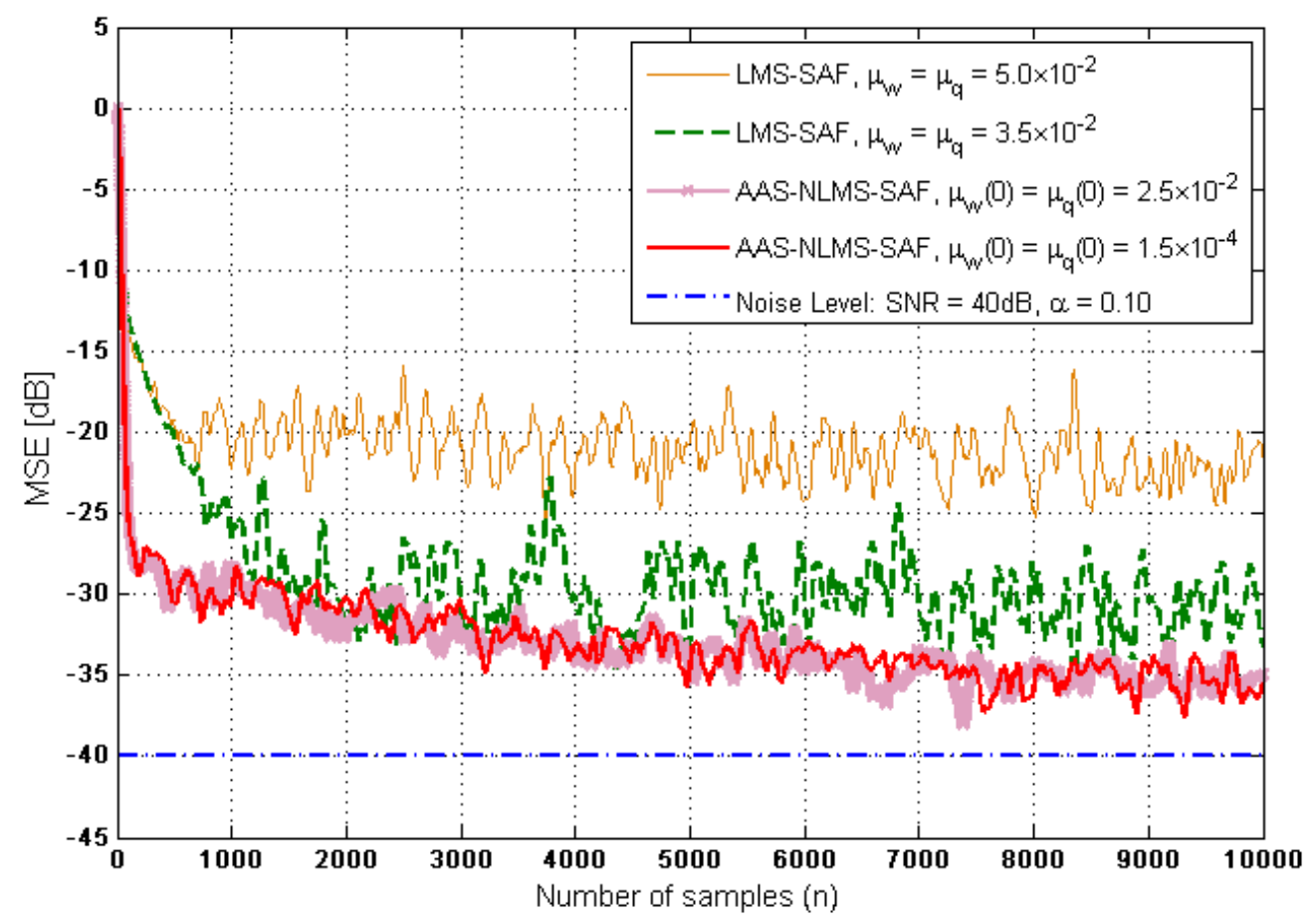

Figure.4 Mean square error (MSE) of proposed ASS-NLMS-SAF algorithm compare with LMS-SAF [20] with the different of initial step-size parameter using SNR $=40 \mathrm{~dB}$ and $\alpha=0.10$ 


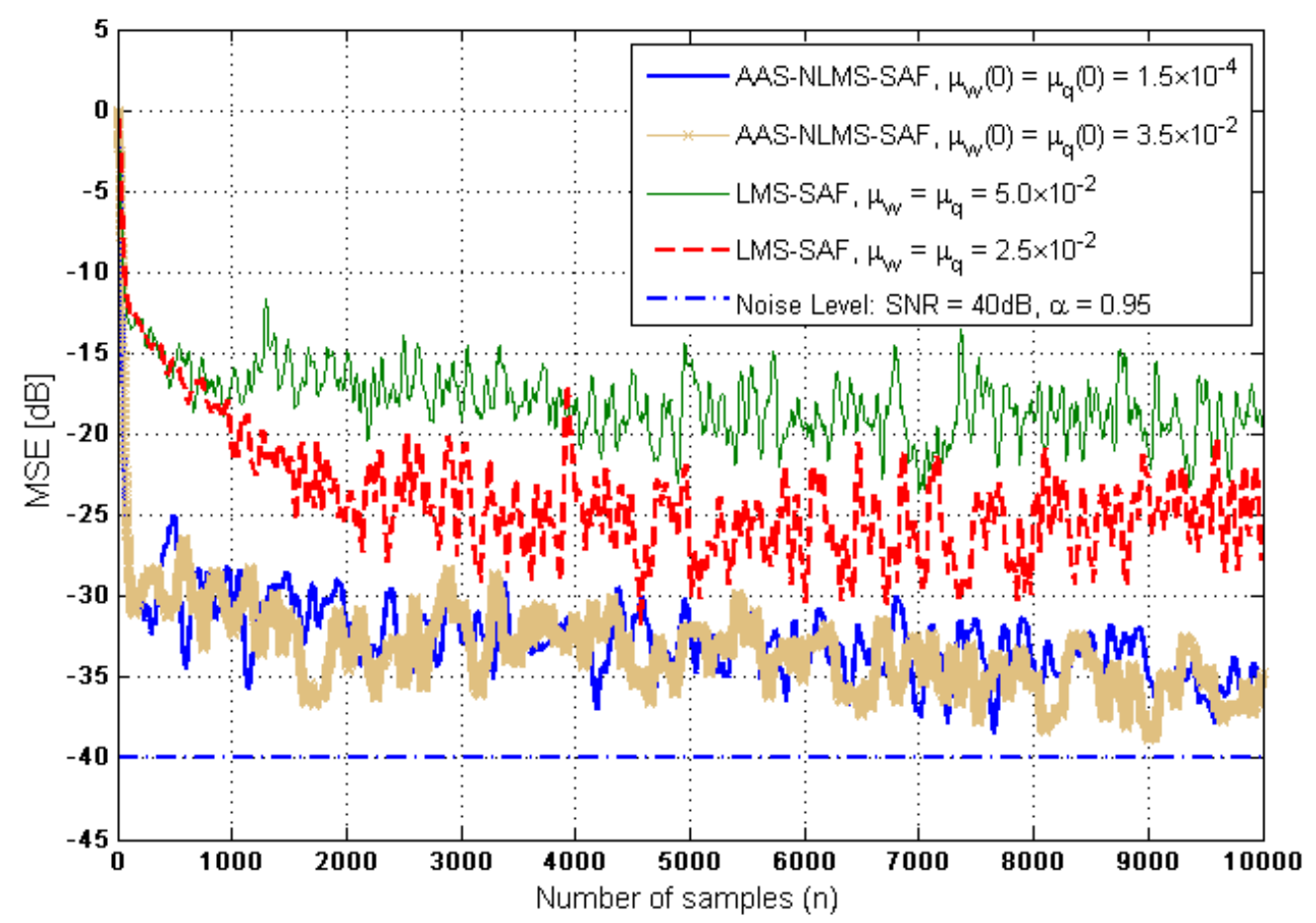

Figure.5 Mean square error (MSE) in dB of proposed ASS-NLMS-SAF algorithm compare with LMS-SAF [20] with the different of initial step-size parameter using SNR $=40 \mathrm{~dB}$ and $\alpha=0.95$

We consider the mean square error (MSE) computed in $\mathrm{dB}$ as

$$
\operatorname{MSE}_{n}=10 \log \left(E\left\{\left(d_{n}-\mathbf{u}_{n}^{\mathrm{T}} \mathbf{C} \mathbf{q}_{i, n}\right)^{2}\right\}\right) .
$$

A 23-point LUT $\boldsymbol{q}_{0}$ is implemented for a nonlinear memoryless target function that is interpolated with a uniform third degree spline and SAF model is used as $\triangle x=0.2$ [4] and $\mathbf{C}$ is a Catmul-Rom spline as described in [2].

Initial parameters of all SAF model are as follows: $\mathbf{w}(0)=\varphi_{w} \cdot[1,0, \ldots, 0]^{\mathrm{T}}$, where $\varphi_{w}=$ $1 \times 10^{-3}, \quad \mathbf{q}(0)=[1,0, \ldots, 0]^{\mathrm{T}}, \quad$ SNR $=40 \mathrm{~dB}$, length of filter is 5 . For initial parameters for spline adaptive filtering based on least mean square (LMSSAF) algorithm [20] are as: $\mu_{w}=\mu_{q}=0.025$, $0.035,0.050$. Summary of LMS-SAF is shown in Table 2.

Other parameters for proposed AAS-NLMSSAF algorithm are as: $\mu_{w}(0)=\mu_{q}(0)=1.5 \times 10^{-4}$, $1.5 \times 10^{-2}, 2.5 \times 10^{-2}, 3.5 \times 10^{-2}, 5.5 \times 10^{-2}$. The fixed parameters are as follows: $\alpha_{w}=\alpha_{q}=0.975$, $\beta_{w}=2.95 \times 10^{-3}, \beta_{q}=1.95 \times 10^{-3}, \gamma=0.97$.

Learning rates of step-size parameters $\mu_{w_{n}}$ of tap-weight vector and $\mu_{q_{n}}$ of control points vector of proposed AAS-NLMS-SAF algorithm are shown in Figs. 2 and 3 with the different initial parameter of
Table 2. Spline adaptive filter based on the least mean square algorithm (LMS-SAF) [20]

Initialize $: \mathbf{w}(0)=\mathbf{q}(0)=\varphi_{w^{\cdot}}\left[\begin{array}{lll}1 & 0 & \ldots .0\end{array}\right]^{T}$ for $n=0,1,2, \ldots, N-1$.

1) To determine the tap-weight vector $\mathbf{w}_{n}$

$$
\mathbf{w}_{n+1}=\mathbf{w}_{n}+\mu_{w} \mathbf{u}_{n}^{\prime} \mathbf{C} \mathbf{q}_{i, n} \mathbf{x}_{n} e_{n}
$$

2) To determine the tap-weight vector $\mathbf{w}_{n}$ and the control point vector $\mathbf{q}_{i, n}$ as

$$
\mathbf{q}_{i, n+1}=\mathbf{q}_{i, n}+\mu_{q} \mathbf{C}^{T} \mathbf{u}_{n} e_{n}
$$

end

$\mu_{w}(0), \mu_{q}(0)$ at $\mathrm{SNR}=40 \mathrm{~dB}$ with the different $\alpha$ in (60) generated the input coloured signal. It is seen that both learning curves of $\mu_{w_{n}}$ and $\mu_{q_{n}}$ converge to their equilibria despite 100 -fold of initial step-size situations at steady-state.

In terms of MSE performance, simulation results shown for the proposed experiments with the two choices of parameter $\alpha=0.10,0.95$ which are presented in Fig. 4 and Fig. 5, respectively. At steady-state, the performance of proposed AASNLMS-SAF algorithm closes to the noise power. In 
addition, we notice that the performance of proposed AAS-NLMS-SAF algorithm outperforms to converge faster and robust mechanism when compared with the LMS-SAF algorithm using the variants of fixed step-size parameter.

\section{Conclusion}

In this paper, we propose a step-size approach in term of averaging of square error for spline adaptive filtering (AAS-NLMS-SAF). We describe how to derive the proposed adaptive averaging step-size algorithm with the method of normalised version of LMS algorithm on spline adaptive filtering. By using an estimation of autocorrelation between present estimated error and a priori estimated error, the adaptive averaging step-size scheme is proposed on SAF. The convergence and stability analysis of proposed AAS-NLMS-SAF algorithm examine in terms of mean square error and excess mean square error concerned with adaptive tap-weight FIR vector and control points vector in the adaptive LUT.

Both the trajectories of adaptive step-size parameters can converge into each equilibrium in spite of 100-fold initial variations. Learning curves of MSE performance are illustrated to converge dramatically to steady-state in comparison with the existing LMS-SAF algorithm using the fixed stepsize parameters.

Especially, SAF can perform well with low-cost complexity beside the existing FIR structures. Because of the recursion form, SAF can be modified in many practical cases such as nonlinear channel equalization, biomedical data analysis and control applications.

\section{References}

[1] A. Uncini, "Fundamentals of Adaptive Signal Processing", ser. Signals and Communication Technology, Springer International Publishing, Switzerland, 2015.

[2] M. Scarpiniti, D. Comminiello, R. Parisi, and A. Uncini, "Nonlinear Spline Adaptive Filtering”, Signal Processing, Vol. 93, Issue. 4, pp. 772-783, 2013.

[3] C. Liu, Z. Zhang, and X. Tang, "Sign Normalised Spline Adaptive Filtering Algorithms Against impulsive Noise", Signal Processing, Vol. 148, Issue. 6, pp. 234-240, 2018.

[4] L. Ljung, "System Identification- Theory for the user", Upper Saddle River, NJ, 1999.

[5] M. Scarpiniti, D. Comminiello, R. Parisi, and A. Uncini, "Hammerstein Uniform Cubic Spline Adaptive Filtering: Learning and
Convergence Properties", Signal Processing, Vol. 100, pp. 112-123, 2014.

[6] M. Scarpiniti, D. Comminiello, R. Parisi, and A. Uncini, "Novel Cascade Spline Architectures for the Identification of Nonlinear Systems", IEEE Transactions on Circuits and Systems I: Regular Papers, Vol. 62, No. 7, pp. 1825-1835, 2015.

[7] S. Scardapane, M. Scarpiniti, D. Comminiellow, and A. Uncini, "Diffusion Spline Adaptive Filtering", In: Proc. of European Signal Processing Conference, pp. 1498-1502, 2016.

[8] S. Guan and Z. Li, "Normalised Spline Adaptive Filtering Algorithm for Nonlinear System Identification", Neural Processing Letter, V ol. 5, pp.1-13, 2017.

[9] S. Guan and Z. Li, "Normalised Spline Adaptive Filtering Algorithm for Nonlinear System Identification", Neural Processing Letter, Vol. 46, Issue. 2, pp. 595-607, 2017.

[10] M. Scarpiniti, D. Commiiniello, R. Parisi, and A. Uncini, "Nonlinear System Identification using IIR Spline Adaptive Filters", Signal Processing, Vol. 108, pp. 30-35, 2015.

[11] C. Liu and Z. Zhang, "Set-membership Normalised Least $M$-estimate Spline Adaptive Filtering Algorithm in Impulsive Noise", Electronics Letters, Vol. 54, No. 6, pp. 393395, 2018.

[12] C. Liu, Z. Zhang, and X. Tang, "Sign Normalised Spline Adaptive Filtering Algorithms Against Impulsive Noise", Signal Processing, Vol. 148, pp. 234-240, 2018.

[13] H.S. Lee, S.E. Kim, W. Lee, and W.J. Song, "A Variable Step-size Diffusion LMS algorithm for Distributed Estimation", IEEE Transactions on Signal Processing, Vol. 63, No. 7, pp. 18081820, 2015.

[14] S. Sitjongsataporn, "Advanced Adaptive DMT Equalisation: Algorithms and Implementation", LAP LAMBERT Academic Publishing, 2011.

[15] L. Wang, Y. Cai, and R.C.de Lamare, "LowComplexity Adaptive Step- Size Constrained Constant Modulus SG-based Algorithms for Blind Adaptive Beamforming", In: Proc. of International Conference on Acoustics, Speech, and Signal Processing, pp. 2593-2596, 2008.

[16] S. Sitjongsataporn and P. Yuvapoositanon, "Low Complexity Adaptive Step-Size Filtered Gradient-based Per-Tone DMT Equalisation", In: Proc. of International Symposium on Circuits and Systems, pp.2526-2529, 2010.

[17] S. Kalluri and G.R. Arce, "General Class of Nonlinear Normalized Adaptive Filtering Algorithms", IEEE Transactions on Signal 
Processing, Vol. 48, No. 8, pp. 2262-2272, 1999.

[18] S. Guarnieri, F. Piazza, and A. Uncini, "Multilayer Feedforward Networks with Adaptive Spline Activation Function", IEEE Transactions on Neural Network, Vol. 10, No. 3, pp. 672-683, 1999.

[19] S. Sitjongsataporn, "Analysis of Low Complexity Adaptive Step-size Orthogonal Gradient-based FEQ for OFDM systems", ECTI Transactions on Computer and Information Technology, Vol. 5, No. 2, pp. 134145, 2011.

[20] M. Scarpiniti, D. Comminiello, R. Parisi, and A. Uncini, "Spline Adaptive Filters: Theory and Applications", Adaptive Learning Methods for Nonlinear System Modelling, pp. 47-69, 2018. 